\title{
Theoretical and Experimental Analysis of Energy in Charging a Capacitor by Step-Wise Potential
}

\author{
Sami M. Al-Jaber, Iyad Saadeddin* \\ Department of Physics, An-Najah National University, Nablus, Palestine \\ Email: jaber@najah.edu, ${ }^{\star I y a d s @ n a j a h . e d u ~}$
}

How to cite this paper: Al-Jaber, S.M. and Saadeddin, I. (2020) Theoretical and Experimental Analysis of Energy in Charging a Capacitor by Step-Wise Potential. Journal of Applied Mathematics and Physics, 8, 38-52.

https://doi.org/10.4236/jamp.2020.81004

Received: October 18, 2019

Accepted: December 28, 2019

Published: December 31, 2019

Copyright $\odot 2020$ by author(s) and Scientific Research Publishing Inc. This work is licensed under the Creative Commons Attribution International License (CC BY 4.0).

http://creativecommons.org/licenses/by/4.0/

\begin{abstract}
In this paper, charging capacitor in RC circuit, to a final voltage, via arbitrary number of steps, is investigated and analyzed both theoretically and experimentally. The obtained results show that the stored energy in the capacitor is constant independent of $N$, but the dissipated energy in the resistor and the consumed energy by the power supply decreases as number of steps $N$ increases (adiabatic charging). The limit when the step number goes to infinity is examined and our result shows that the dissipated energy vanishes theoretically. This limit is carried out experimentally by using a ramp potential.
\end{abstract}

\section{Keywords}

Charging Capacitor, RC Circuit, Stored Energy, Dissipated Energy, Adiabatic Charging

\section{Introduction}

Charging and discharging capacitors play important role in many applications of electrical energy storage [1] [2] [3]. During the charging process of a capacitor, the energy dissipation is usually of great concern [4] [5] [6] [7], so the efficient charging is a crucial factor and has been recently investigated [8] [9] [10]. Furthermore, reduction in energy dissipation has become one of the major concerns in communications and computing [11] [12] [13]. Recently, there has been more concern about energy storage and dissipation with the emergence of Graphene and other nanomaterials in recent development of supercapacitors [14] [15] [16] [17]. The usual treatment of charging a capacitor is the traditional $R$ - $C$ circuit, in which a capacitor of capacitance $c$ and resistor of resistance $R$ are connected in series with a power supply of constant voltage, $V_{0}$. It is widely well-known that 
the dissipated heat energy, $Q$ in the resistor is equal to the energy stored, $U$ in the capacitor when it is finally fully charged, namely

$$
U=Q=\frac{1}{2} c V_{0}^{2} \text {. }
$$

Therefore, the consumed energy by the power supply is divided equally between the stored energy in the capacitor and the dissipated energy regardless of the value of the resistance, $R$. It has long been recognized that abruptly charging the capacitor is accompanied by energy loss given by Equation (1). However, workers suggested gradual (adiabatic) charging to lower the energy loss [18] [19] [20] [21].

In this paper, we consider $R C$ circuit in which the capacitor is charged up to a final potential $V_{0}$ through $N$ steps. We derive the energy stored, the dissipation energy, and the consumed energy at the end of arbitrary $f^{\text {th }}$ step. We also setup an experiment for this adiabatic charging and compare the theoretical derived quantities with the experimental ones. It is shown that, the final energy stored in the capacitor is a conserved quantity (given by Equation (1)), but the dissipated energy and the consumed energy by the power supply decrease as the number of steps increases. We also consider, theoretically, the special case, $N \rightarrow \infty$, in which case the dissipation energy vanishes and the consumed energy is just the energy stored in the capacitor. Experimentally, this special case is considered by using a ramp potential in the $R C$ circuit and we show that the experimental results are in good agreement with the theoretical ones.

The structure of the manuscript is as follows: In Section 2, charging a capacitor in $R C$ circuit using stepwise potential is theoretically examined and analyzed, so as the dissipation energy and the consumed energy are expressed in terms of the number of steps. In Section 3, the charging process of the capacitor in $R C$ circuit is carried out experimentally using $N$ steps potential, so the final charge on the capacitor is measured as the area under the current-time curve for each $N$. This enables us to compute the stored and the consumed energies, so the dissipation energy is computed from the difference between the two. Section 3 is devoted for conclusions and discussion.

\section{2. $R C$ with $N$ Steps Charging: Theoretical Analysis}

We consider $R C$ in which a capacitor of capacitance $c$ is connected in series to a resistor of resistance $R$ and a power supply. The procedure is to charge the capacitor to a final potential $V_{0}$ through $N$ steps. So in the process, the potential is carried out as

$$
V=\frac{V_{0}}{N}, \frac{2 V_{0}}{N}, \frac{3 V_{0}}{N}, \cdots, \frac{j V_{0}}{N}, \cdots, V_{0} .
$$

So considering the $f^{\text {th }}$ step, the potential of the power supply is $j V_{0} / N$ and thus Kirchhoff law yields

$$
j \frac{V_{0}}{N}-\frac{q}{c}-i R=0,
$$

whose solution gives the charge on the capacitor during the $f^{\text {th }}$ step; 


$$
q_{j}(t)=\frac{V_{0} c}{N}\left[j-\mathrm{e}^{-t / R c}\right] .
$$

So the final charge at the end of the $f^{t h}$ step is $q_{j}=\frac{j V_{0} c}{N}$. It is noticed that the amount of charge during each step is the same, which can be seen from $q_{j}-q_{j-1}$ which is clearly equals to $V_{0} c / N$.

The heat energy dissipated in the resistor during the $f^{\text {th }}$ step is:

$$
Q_{\text {during } j}=\int_{0}^{\infty} i^{2} R \mathrm{~d} t=R \int_{0}^{\infty}\left(\frac{V_{0}}{N R}\right)^{2} \mathrm{e}^{-2 t / R c} \mathrm{~d} t=\frac{1}{2} c \frac{V_{0}^{2}}{N^{2}},
$$

where the current, $i=\frac{\mathrm{d} q_{j}}{\mathrm{~d} t}$ is found from Equation (4). It is clear that the dissipated heat energy is independent of the step number, and thus it is the same for each step. Therefore, the accumulated dissipated heat energy at the end of the $f^{\text {th }}$ step, $Q_{j}$ is

$$
Q_{j}=j Q_{\text {during } j}=\frac{1}{2} c \frac{V_{0}^{2}}{N^{2}} j,
$$

and therefore, at the end of the final step $(j=N)$, the total dissipated heat energy is given by

$$
Q=\frac{1}{2} c \frac{V_{0}^{2}}{N} .
$$

This shows that $Q$ decreases as $N$ increases and thus the dissipated heat energy vanishes as $N \rightarrow \infty$.

Now we consider the energy stored in the capacitor during this process:

At the end of the $f^{\text {th }}$ step, the potential $V_{j}=j V_{0} / N$, so the stored energy is

$$
U_{j}=\frac{1}{2} c V_{j}^{2}=\frac{1}{2} c\left(\frac{j V_{0}}{N}\right)^{2} .
$$

This can be proved by mathematical induction as follows;

1) For $j=1, U_{1}=\int V \mathrm{~d} q=\int_{q_{0}}^{q_{1}} \frac{q}{c} \mathrm{~d} q=\left.\frac{1}{2 c} q^{2}\right|_{q_{0}} ^{q_{1}}$.

But, $q_{0}=0$ and $q_{1}=\frac{V_{0}}{N} c$. Therefore $U_{1}=\frac{1}{2} c\left(\frac{V_{0}}{N}\right)^{2}$.

2) We assume it is true at $j$ and need to show it is true at $j+1$.

Energy stored between steps $j$ and $j+1$ is

$$
U_{j, j+1}=\int_{q_{j}}^{q_{j+1}} \frac{q}{c} \mathrm{~d} q=\frac{1}{2} c\left(\frac{V_{0}}{N}\right)^{2}\left[(j+1)^{2}-j^{2}\right] .
$$

But the stored energy at end of the $(j+1)^{t h}$ step is

$$
\begin{gathered}
U_{j+1}=U_{j}+U_{j, j+1}=\frac{1}{2} c\left(\frac{V_{0}}{N}\right)^{2}\left[j^{2}+(j+1)^{2}-j^{2}\right] . \\
\text { So, } U_{j+1}=\frac{1}{2} c\left(\frac{V_{0}}{N}\right)^{2}(j+1)^{2} .
\end{gathered}
$$


That completes the proof, therefore the energy stored in the capacitor at the end of the $f^{\text {th }}$ step is: $U_{j}=\frac{1}{2} c\left(\frac{V_{0} j}{N}\right)^{2}$, So that when $j=N$, it gives $U=\frac{1}{2} c V_{0}^{2}$, which shows that the energy stored in the capacitor is a conservative quantity, i.e it depends on the potential difference between the initial and final states. It is interesting to note, by comparing Equation (6) and Equation (8), that at the end of the $f^{\text {th }}$ step $U_{j} \geq Q_{j}$. The equality sign holds only for $j=1$.

Finally, we consider the consumed energy by the power supply (input energy):

This can be achieved by two methods: In the first, we calculate the work done by the power supply throughout the whole process and, in the second, we add the energy stored in the capacitor to the dissipation energy.

For the first method: We compute the input energy for each step and then we sum to the required step (say the $f^{\text {th }}$ step). Letting $E_{i-1, i}$ be the input energy between the $(i-1)^{t h}$ step and the $i^{\text {th }}$ step, one may write

$$
E_{i-1, i}=q_{i-1, i} V_{i}=\left(\frac{c V_{0}}{N}\right)\left(\frac{i V_{0}}{N}\right)=\frac{c V_{0}^{2}}{N^{2}} i
$$

Therefore, the input energy, $E_{j}$ up to the $f^{\text {th }}$ step is given by

$$
E_{j}=\sum_{i=0}^{j} E_{i-1, i}=\frac{c V_{0}^{2}}{N^{2}} \sum_{i=0}^{j} i=\frac{c V_{0}^{2}}{N^{2}} \frac{j(j+1)}{2} .
$$

The total input energy, $E$ is obtained by substituting, $j=N$, with the result;

$$
E_{j}=\frac{c V_{0}^{2}}{2} \frac{N+1}{N}
$$

Obviously, in the limit $N \rightarrow \infty$, Equation (11) gives $E=\frac{1}{2} c V_{0}^{2}$ which is equal to the energy stored in the capacitor. This is expected since, in this case, the dissipation energy vanishes.

For the second method: At the end of the $f^{t h}$ step, the input energy is the sum of the energy stored in the capacitor and the dissipation energy, which with the help of Equation (6) and Equation (8), one gets

$$
E_{j}=U_{j}+Q_{j}=\frac{1}{2} c\left(\frac{V_{0} j}{N}\right)^{2}+\frac{1}{2} c \frac{V_{0}^{2}}{N^{2}} j .
$$

The above equation can be simply written as

$$
E_{j}=\frac{1}{2} c \frac{V_{0}^{2}}{N^{2}} j(j+1),
$$

which is exactly the same as Equation (10). It reproduces the total input energy, given by Equation (11), when $j=N$.

\section{3. $R C$ with $N$ Steps Charging: Experimental}

In this section, we consider $R C$ circuit with applied voltage that can be set at any number of steps, $N$ to achieve a final voltage $V_{0}$. The potential steps was applied 
using computerized PGZ 402 universal potentiostate (radiometer analytical). Connection to $R C$ circuit was established using two electrode connections mode as shown in Figure 1.

The procedure goes as follows: The voltage source is set at the required number of steps with duration time for each step much greater than the time constant (RC) of the circuit. The current in the circuit is plotted as function of time. The total charge, $q$ delivered by the power source and accumulated at the capacitor is calculated by finding the area under the current-time curve. The final energy stored in the capacitor is calculated from the formula, $U=q^{2} / 2 c$. The input energy (consumed energy) is calculated as follows:

$$
E=q V_{\text {avg }} \text {, }
$$

where $V_{\text {arg }}$ is the average potential the process of $N$ steps and is given by

$$
V_{\text {avg }}=\frac{1}{N} \sum_{j=1}^{N} \frac{V_{0}}{N} j=\frac{V_{0}}{N^{2}} \sum_{j=1}^{N} j .
$$

Using $\sum_{j=1}^{N} j=N(N+1) / 2$, Equation (14) becomes

$$
V_{\text {avg }}=V_{0} \frac{N+1}{2 N},
$$

so the input energy becomes

$$
E=q V_{0} \frac{N+1}{2 N} .
$$

The dissipated energy is calculated from the difference between $E$ and $U$, namely

$$
Q=E-U=q V_{0} \frac{N+1}{2 N}-\frac{q^{2}}{2 c} .
$$

The experimental measurements were taken for different values of the number of steps, $N$. The special case $N \rightarrow \infty$ was considered by applying a ramp potential from the power source. For each value of $N$, a graph for current vs. time is obtained and these graphs are shown in Figures 2-12. Each contains the stepwise potential for the given value of the step number, $N$. Figure 2 , for $N=1$, represents the conventional charging capacitor by a constant voltage source. The current for any value of the step number $N$ can be found from Equation (4), namely

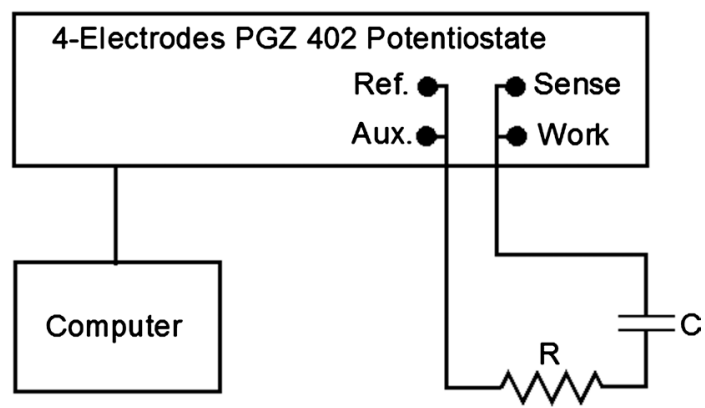

Figure 1. Schematic diagram for RC circuit connected to PGZ 402 Universal Potentiostate using two electrode mode connection. 


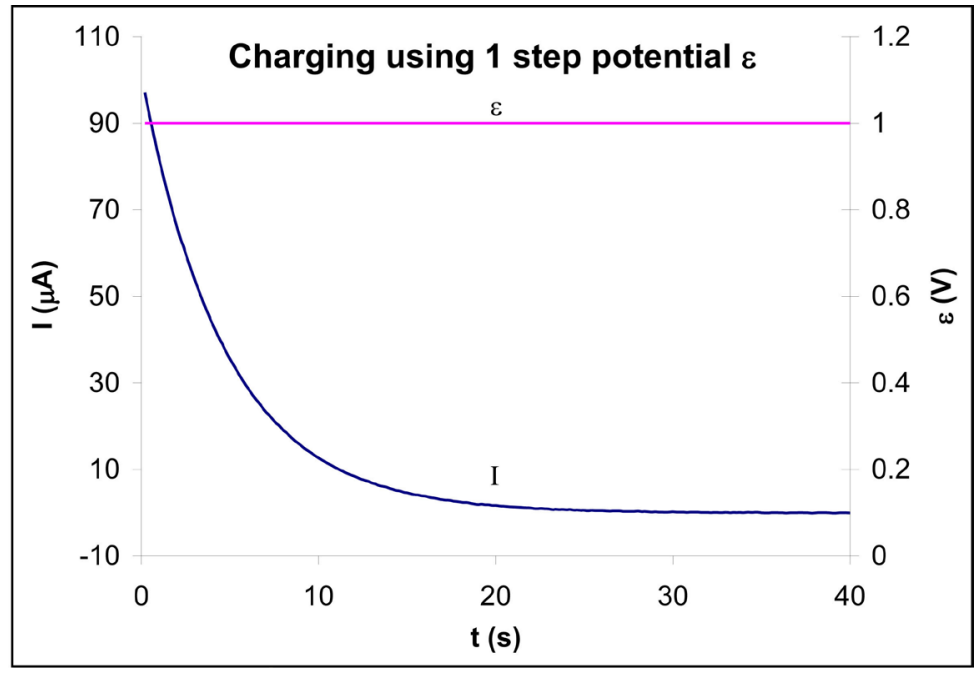

Figure 2. Ivs. $t$ for abrupt potential $(N=1)$.

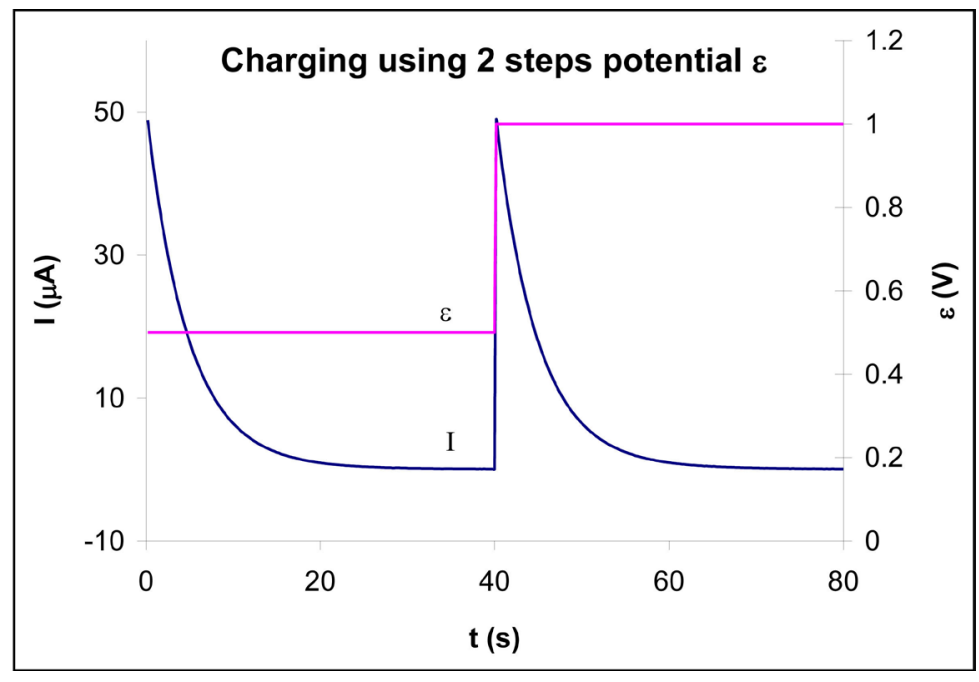

Figure 3. $I$ vs. $t$ for step potential $(N=2)$.

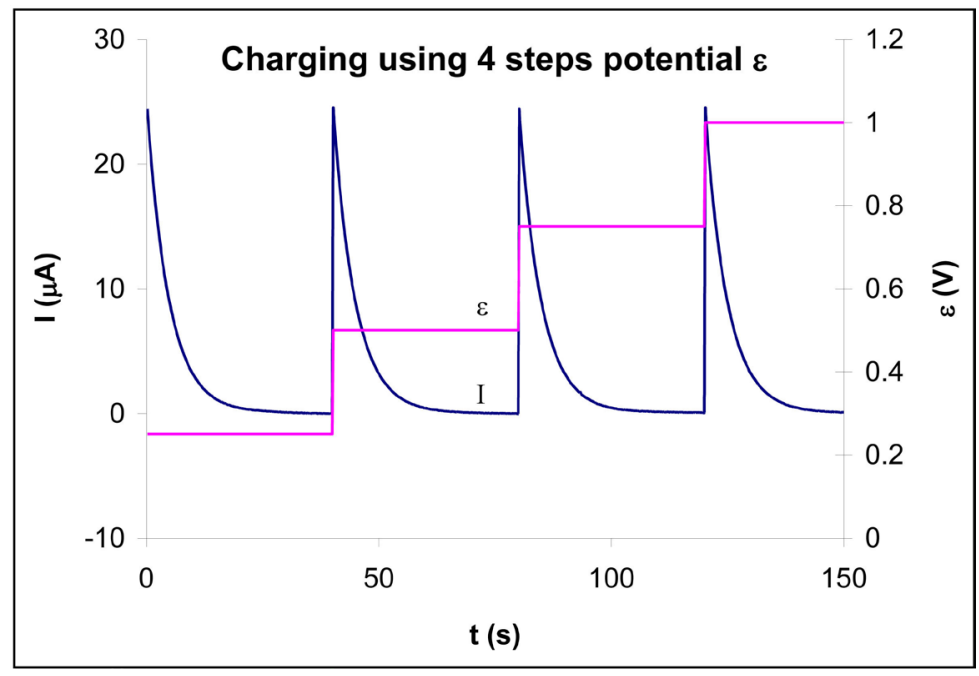

Figure 4. Ivs. $t$ for step potential $(N=4)$. 


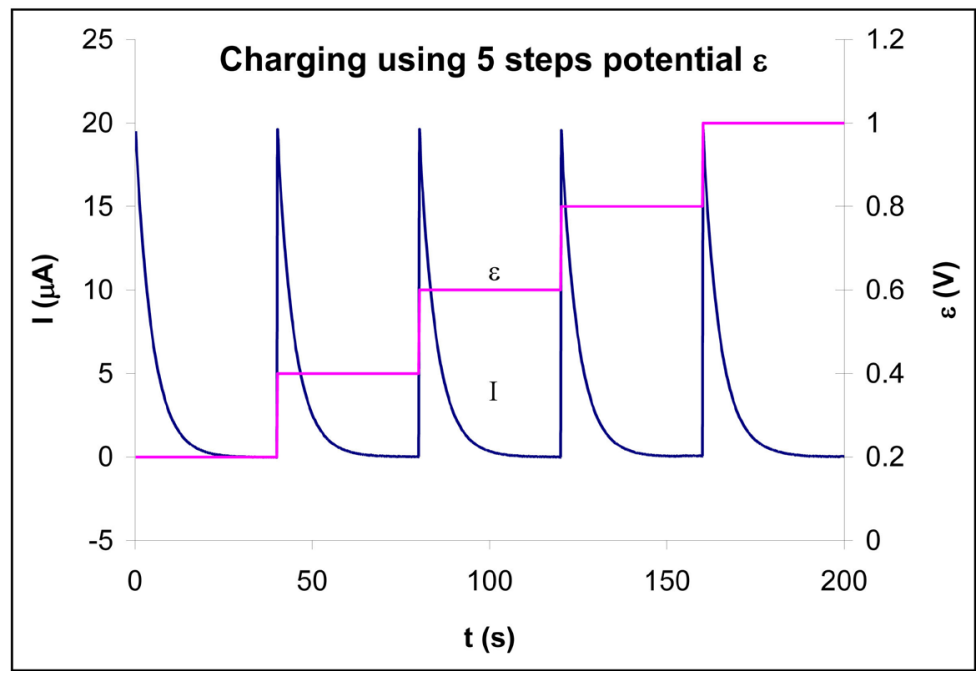

Figure 5. $I$ vs. $t$ for step potential $(N=5)$.

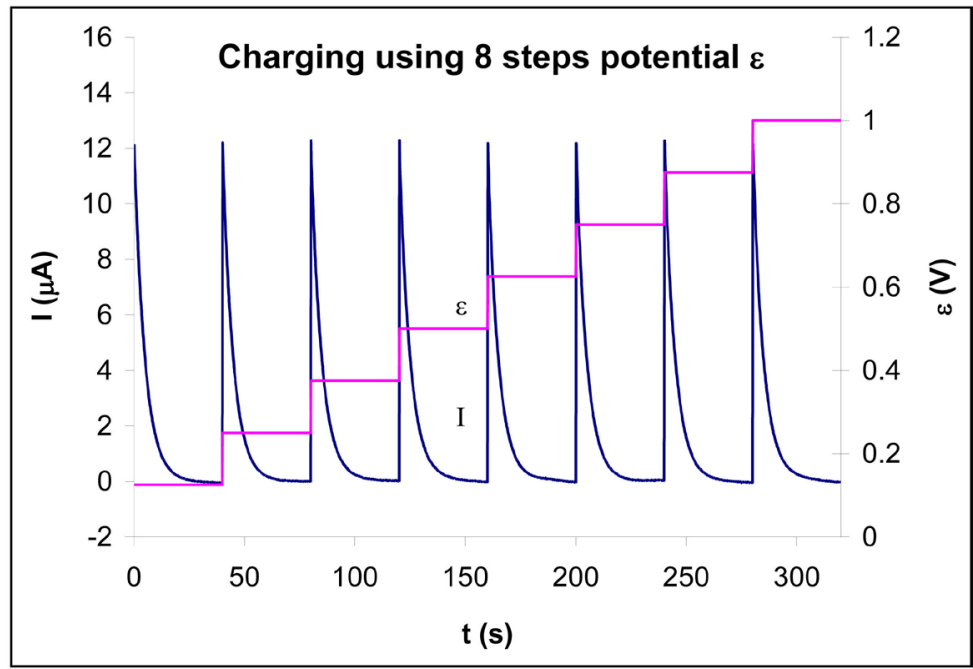

Figure 6. Ivs. $t$ for step potential $(N=8)$.

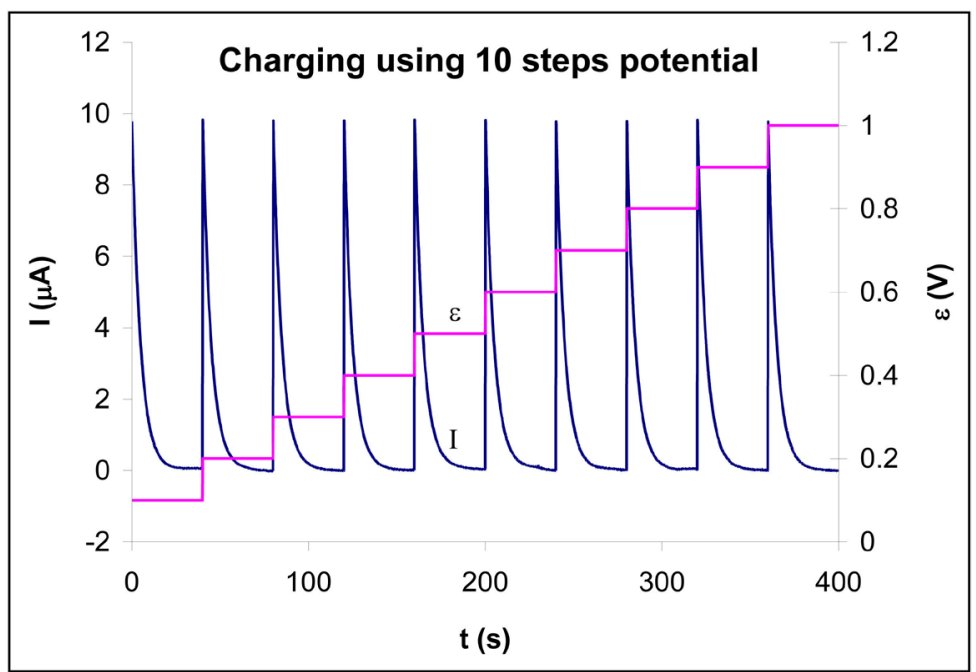

Figure 7. Ivs. $t$ for step potential $(N=10)$. 


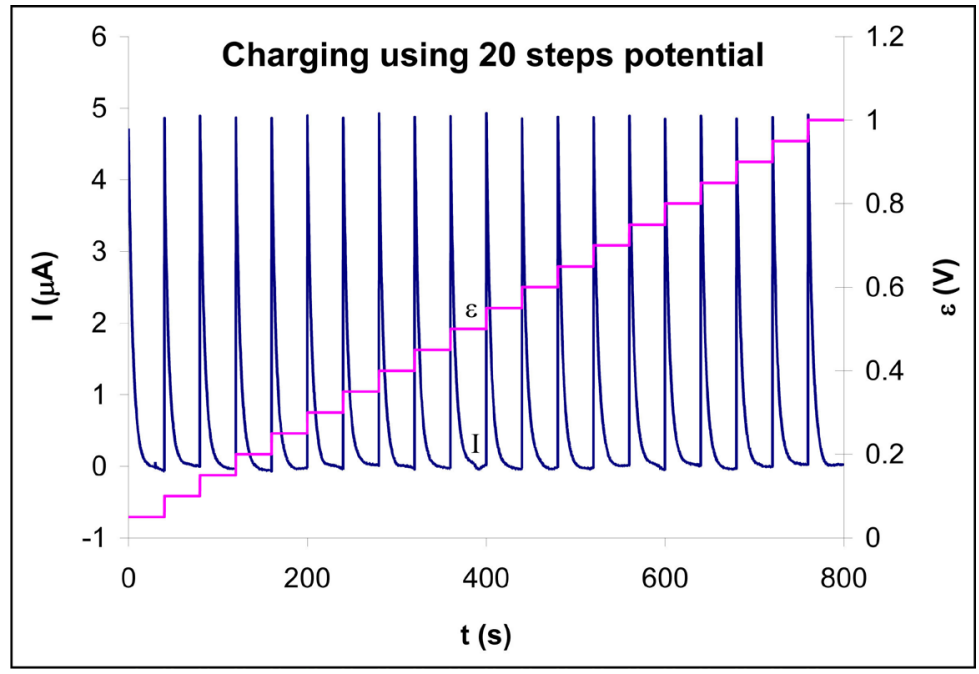

Figure 8. Ivs. $t$ for step potential $(N=20)$.

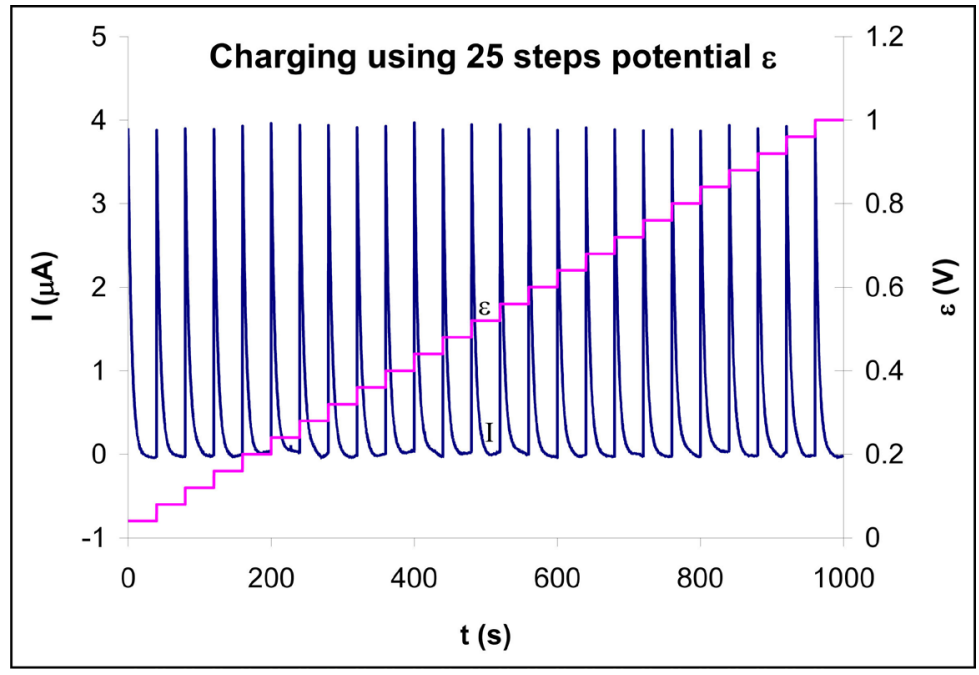

Figure 9. $I$ vs. $t$ for step potential $(N=25)$.

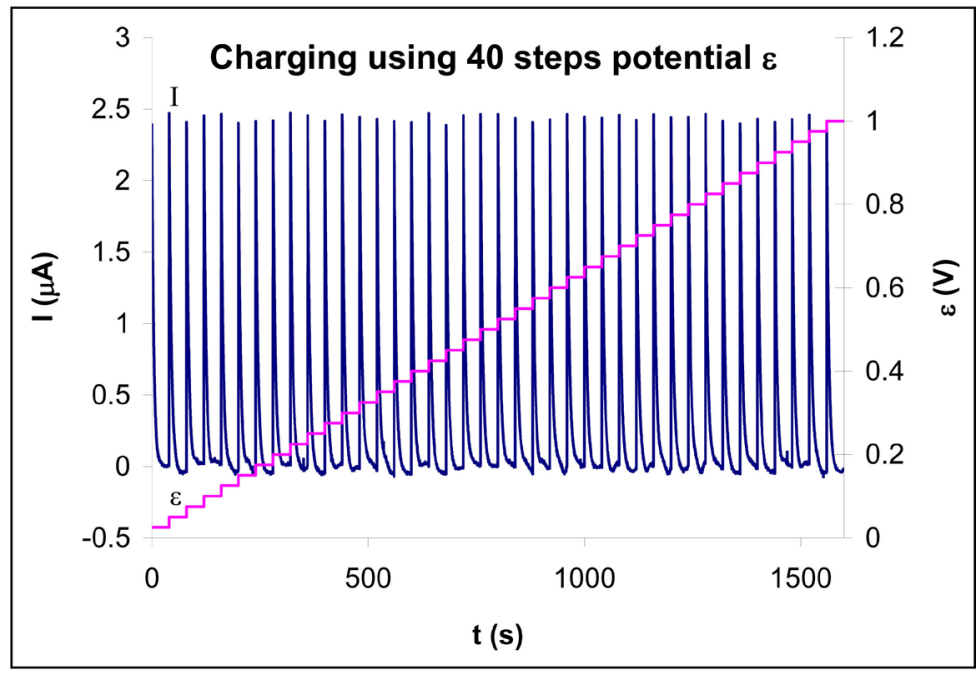

Figure 10. $I$ vs. $t$ for step potential $(N=40)$. 


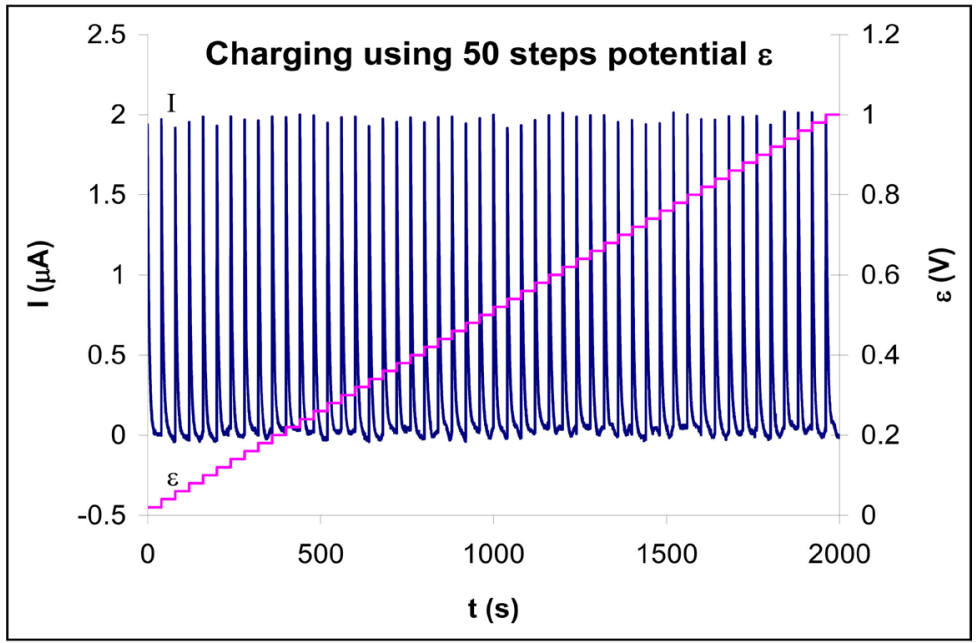

Figure 11. Ivs. $t$ for step potential $(N=50)$.

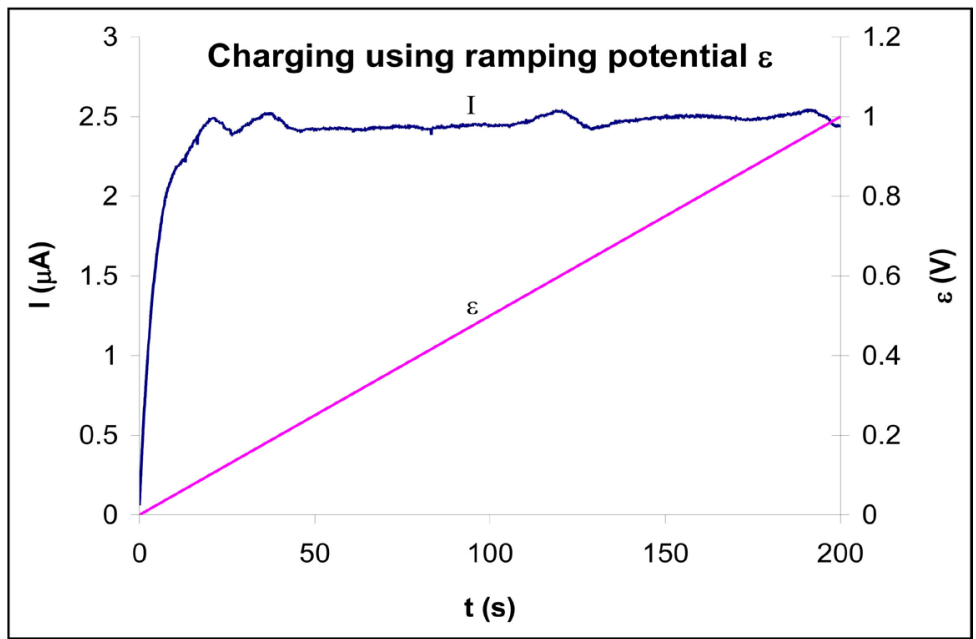

Figure 12. Ivs. $t$ for ramp potential $(N=\infty)$.

$$
i(t)=\frac{\mathrm{d} q}{\mathrm{~d} t}=\frac{V_{0}}{R N} \mathrm{e}^{-t / R c} .
$$

At the beginning of each step $(t=0)$, the initial current, $i_{0}=V_{0} / R N$. Using the values, $V_{0}=1 \mathrm{~V}$, and $R=10^{4} \Omega$, the initial current for each step becomes $i_{0}=\frac{100}{N} \mu \mathrm{A}$. This value is in agreement with the experimental value for each step number in all stepwise potentials. Figure 12 represents the current-time curve for the ramp potential which corresponds to the special stepwise case, $N \rightarrow \infty$. In order to explain this case, we derive below the current as function of time when the power source is a ramp potential. Consider the ramp potential $V(t)=\frac{V_{0}}{\tau} t$, where $\tau(=R c)$ is the time constant of the $R C$ circuit. Applying Kirchhoff law to the circuit gives us

$$
\frac{q}{c}+i R=\frac{V_{0}}{\tau} t
$$


which can be written as

$$
\frac{\mathrm{d} q}{\mathrm{~d} t}+\frac{1}{R c} q=\frac{V_{0}}{R \tau} t .
$$

The above equation is a linear, inhomogeneous, first-order differential equation, which has the same structure as the standard form, namely

$$
\frac{\mathrm{d} x}{\mathrm{~d} t}+p(t) x=f(t) .
$$

The well-known method to solve the above equation is by the integrating factor method. In this method, defining an integrating factor $u(t)=\mathrm{e}^{\int p(t) \mathrm{d} t}$, then the solution for Equation (21) is

$$
x(t)=\frac{1}{u(t)}\left(\int u(t) f(t) \mathrm{d} t+A\right),
$$

where $A$ is a constant to be found from the initial condition. Applying this method to our differential Equation (21), we get $u(t)=\mathrm{e}^{t / R c}, f(t)=\frac{V_{0}}{R \tau} t$, so that the integral in Equation (22), gives (after integration by parts)

$$
\int \frac{V_{0}}{R \tau} t \mathrm{e}^{t / R c} \mathrm{~d} t=\frac{V_{0}}{R \tau}\left[R c t-(R c)^{2}\right] \mathrm{e}^{t / R c} .
$$

Therefore, with $x(t) \rightarrow q(t)$, the solution of Equation (20) is

$$
q(t)=\frac{c V_{0}}{\tau} t-\frac{R V_{0} c^{2}}{\tau}+A \mathrm{e}^{-t / R c} .
$$

Since the capacitor is initially uncharged, so $q=0$ at $t=0$ determines the constant $A$, and the substitution, $\tau=R c$, enables us to write Equation (24) as

$$
q(t)=\frac{V_{0}}{R} t-c V_{0}\left(1-\mathrm{e}^{-t / R c}\right) .
$$

The electric current is now readily obtained, using $i=\mathrm{d} q / \mathrm{d} t$, with the result of

$$
i=\frac{V_{0}}{R}\left(1-\mathrm{e}^{-t / R c}\right)
$$

Therefore, after a long time $t \gg \tau$, the electric current attains a constant value, $V_{0} / R$. This explains the plateau behavior in Figure 12. The almost linear behavior of the current for very short time $t \ll \tau$ in Figure 12 can be explained by expanding the exponential in Equation (26) and retaining the first two terms to get $i \rightarrow \frac{V_{0}}{R^{2} c} t$, which is linear in time.

Chrono-coulometry experiment has been performed in order to determine the capacitance best value, $c$ of the used commercial capacitor. In this method, a constant voltage, $V_{0}=1 \mathrm{~V}$ is applied and then the charge accumulated on the capacitor is plotted as function of time as shown in Figure 13.

The maximum stored charge in the capacitor, $q=478.24 \mu \mathrm{C}$ (measured when the current is close to zero as the area under the corresponding current-time 
curve. Therefore, the capacitance, $c=\frac{q}{V_{0}}=\frac{478.24 \mu \mathrm{C}}{1 \mathrm{~V}}=478.24 \mu \mathrm{F}$. The experimental and theoretical values of the stored energy are respectively

$$
U_{\exp }=\frac{q^{2}}{2 c}, U_{t h}=\frac{V_{0}^{2}}{2 C},
$$

while the experimental and theoretical values of the dissipated energy and the input energy are calculated from Equations ((17), (7), (16), and (11)) respectively. Our experimental and theoretical results are tabulated in Table 1.

Our results show a good agreement between the experimental and theoretical values. For comparison purposes, we compute the ratios between both the stored energy and the dissipated energy on one hand, and the input energy on the other for the experimental and the theoretical values. These quantities are shown in Table 2.

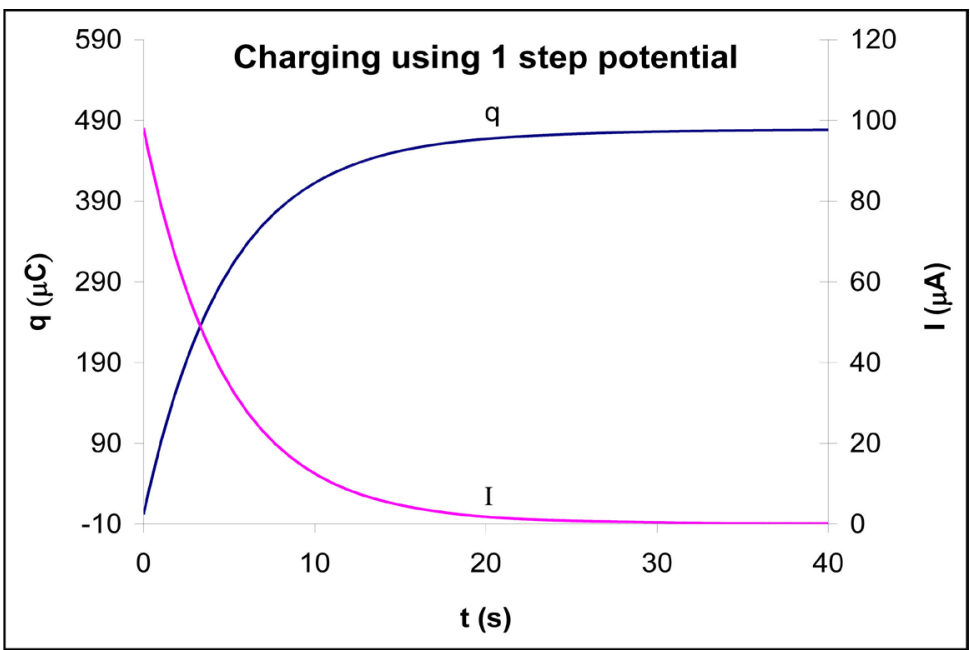

Figure 13. The charge and current as function of time to find the capacitance, $c$.

Table 1. Experimental $(\exp )$ \& theoretical $(t h)$ results for input energy $(E)$, stored energy $(U)$, and dissipated energy $(Q)$ for different values of the step number $N$.

\begin{tabular}{cccccccc}
\hline$N$ & $q(\mu \mathrm{C})$ & $E_{\exp }(\mu \mathrm{J})$ & $E_{t h}(\mu \mathrm{J})$ & $U_{\exp }(\mu \mathrm{J})$ & $U_{t h}(\mu \mathrm{J})$ & $Q_{\exp }(\mu \mathrm{J})$ & $Q_{\text {th }}(\mu \mathrm{J})$ \\
\hline 1 & 478.24 & 478.24 & 478.12 & 239.12 & 239.12 & 239.12 & 239.12 \\
2 & 481.2 & 360.90 & 358.68 & 242.09 & 239.12 & 118.81 & 119.56 \\
4 & 478.3 & 298.93 & 298.90 & 239.18 & 239.12 & 59.75 & 59.78 \\
5 & 487.44 & 292.46 & 286.94 & 248.41 & 239.12 & 44.05 & 47.82 \\
8 & 477.17 & 268.41 & 269.01 & 238.05 & 239.12 & 30.35 & 29.89 \\
10 & 485.77 & 267.17 & 263.03 & 246.71 & 239.12 & 20.47 & 23.91 \\
20 & 463.5 & 243.34 & 251.07 & 224.61 & 239.12 & 18.74 & 11.96 \\
25 & 475.51 & 247.26 & 248.68 & 236.39 & 239.12 & 10.87 & 9.56 \\
40 & 464.86 & 238.24 & 245.10 & 225.93 & 239.12 & 12.31 & 5.98 \\
50 & 482.39 & 246.02 & 243.90 & 243.28 & 239.12 & 2.74 & 4.78 \\
$\infty$ & 481.41 & 240.71 & 239.12 & 242.30 & 239.12 & -1.59 & 0 \\
\hline
\end{tabular}


Table 2. Stored energy and dissipated energy ratios relative to input energy, both experimentally and theoretically.

\begin{tabular}{ccccc}
\hline$N$ & $\left(\frac{U}{E}\right)_{\text {exp }}(\%)$ & $\left(\frac{U}{E}\right)_{\text {th }}(\%)$ & $\left(\frac{Q}{E}\right)_{\text {exp }}(\%)$ & $\left(\frac{Q}{E}\right)_{\text {th }}(\%)$ \\
\hline 1 & 50 & 50 & 50 & 50 \\
2 & 67.1 & 66.7 & 32.9 & 33.3 \\
4 & 80 & 80 & 20 & 20 \\
5 & 84.9 & 83.3 & 15.1 & 16.7 \\
8 & 88.7 & 88.9 & 11.3 & 11.1 \\
10 & 92.3 & 90.9 & 7.7 & 9.1 \\
20 & 92.3 & 95.2 & 7.7 & 4.8 \\
25 & 95.6 & 96.2 & 4.4 & 3.8 \\
40 & 94.8 & 97.6 & 5.2 & 2.4 \\
50 & 98.8 & 98.0 & 1.2 & 2 \\
$\infty$ & 100.6 & 100 & -0.6 & 0 \\
\hline
\end{tabular}

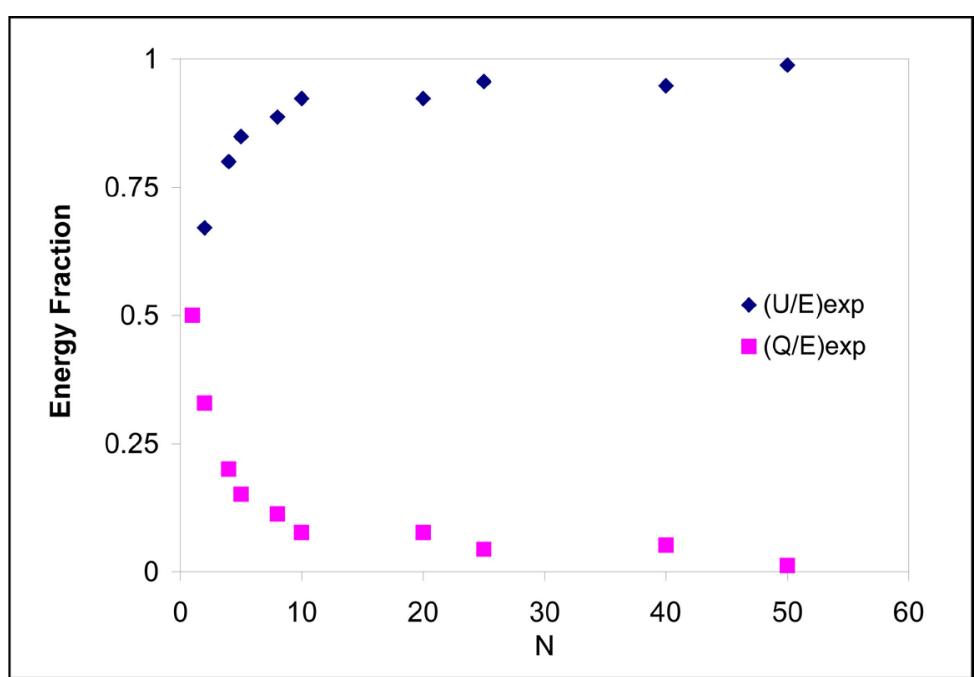

Figure 14. Experimental ratios of both the stored and dissipated energy relative to the input energy.

It is noticed that we achieved a very high percentage for the stored energy ratio, and thus a very low percentage for the dissipation energy ratio. We show in Figure 14, the experimental ratios between the energy stored and the dissipated energy relative to the input energy by the power source.

\section{Discussion and Conclusion}

In this paper, we investigated the stepwise charging of a capacitor in $R C$ circuit. The potential step was applied using the PGZ 402 universal potentiostate. In the first part, theoretical investigation is carried out: Analytical results for the dissipation energy and the consumed energy were derived when charging the capacitor by $N$ steps to achieve the final voltage. It was shown that each of the energy 
dissipation and the consumed energy decreases as the step number $N$ increases, while the energy stored in the capacitor is independent of $N$. An interesting result is that the dissipation energy vanishes as $N \rightarrow \infty$ and in this case the consumed energy is equal to the stored energy in the capacitor. In the second part, experimental analysis is investigated: This has been carried out by plotting current vs. time in each case and the charge on the capacitor is computed from the area under the curve. The calculated charge was used to find the energy stored in the capacitor and the use of Equations (16) and (17) yields the dissipated energy and the consumed energy. Our theoretical results were verified by carrying experiment measurements of the consumed energy, stored energy and the dissipated energy using stepwise potential and the ramp potential that corresponds to the case $N \rightarrow \infty$. Our experimental results were found to be in good agreement with the theoretical ones.

\section{Conflicts of Interest}

The authors declare no conflicts of interest regarding the publication of this paper.

\section{References}

[1] Bezryadin, A., Belkin, A., Llin, E., Pak, M., Colla, E. and Huber, A. (2017) Large Energy Storage Efficiency of the Dielectric Layer of Graphene Nanocapacitors. Nanotechnology, 28, Article ID: 495401. https://doi.org/10.1088/1361-6528/aa935c

[2] Asnawi, R., Nurhadiyanto, A.Z. and Asmara, A. (2018) The Characteristic of Supercapacitors Circuit as a Future Energy Storage Media. Journal of Physics. Conference Series, 1140, Article ID: 012001. https://doi.org/10.1088/1742-6596/1140/1/012001

[3] Fletcher, N.H., Hilton, A.D. and Ricketts, B.W. (1996) Optimization of Energy Storage Density in Ceramic Capacitors. Journal of Physics D: Applied Physics, 29, 253-258. https://doi.org/10.1088/0022-3727/29/1/037

[4] AL-Jaber, S.M. and Salih, S.K. (2000) Energy Consideration in the Two Capacitor Problem. European Journal of Physics, 21, 341-345. https://doi.org/10.1088/0143-0807/21/4/307

[5] Makihara, K., Onoda, J. and Miyakawa, A. (2006) Low Energy Dissipation Electric Circuit for Energy Harvesting. Smart Materials and Structures, 15, 1493-1498. https://doi.org/10.1088/0964-1726/15/5/039

[6] Newburgh, R. (2005) Two Theorems on Dissipative Energy Losses in Capacitor Systems. Physics Education, 40, 370-372. https://doi.org/10.1088/0031-9120/40/4/008

[7] Kahn, M. (1998) Capacitors and Energy Losses. Physics Education, 23, 36-37. https://doi.org/10.1088/0031-9120/23/1/004

[8] Wang, D. (2017) The Most Energy Efficient Way to Charge the Capacitor in a RC Circuit. Physics Education, 52, Article ID: 065019. https://doi.org/10.1088/1361-6552/aa8973

[9] Lee, M. and Kim, J. (2016) Design of a 93\% Energy-Efficient Buck-Type Capacitor Charger IC in 250-nm CMOS. IEEE Transactions on Industry Applications, 52, 3203-3211. https://doi.org/10.1109/TIA.2016.2541086 
[10] Shah, A.S., Arslan, S. and Kim, H. (2018) A Reconfigurable Voltage Converter with Split-Capacitor Charging and Energy Recycling for Ultra-Low-Power Applications. IEEE Access, 6, 68311-68323. https://doi.org/10.1109/ACCESS.2018.2879471

[11] Batygin, K. and Stevenson, D.J. (2010) Inflating Hot Jupiters with Ohmic Dissipation. The Astrophysical Journal Letters, 714, L238-L243. https://doi.org/10.1088/2041-8205/714/2/L238

[12] Roy, K. (2014) Ultra-Low Energy Computing Paradigm Using Giant Spin Hall Devices. Journal of Physics D: Applied Physics, 47, Article ID: 422001.

[13] Plunk, G.G., Tatsuno, T. and Dorland, W. (2012) Considering Fluctuation Energy as a Measure of Gyrokinetic Turbulence. New Journal of Physics, 14, Article ID: 103030.

[14] Palaniselyam, T. and Baek, J. (2015) Graphene Based 2D-Materials for Supercapacitors. 2D Materials, 2, Article ID: 032002.

[15] Bagheri, S., Wu, N. and Filizadeh, S. (2018) Modeling of Capacitor Charging Dynamics in an Energy Harvesting System Considering Accurate Electromechanical Coupling Effects. Smart Materials and Structures, 27, Article ID: 065026.

[16] Shah, R., Zhang, X. and Talapatra, S. (2009) Electrochemical Double Layer Capacitor Eectrodes Using Aligned Carbon Nanotubes Grown Directly on Metals. Nanotechnology, 20, Article ID: 295202.

[17] Tran, C.D., Vu, A.Q. and Van, L.T. (2018) Uniform Coating of Polyaniline on Porous Carbon Nanofibers as Efficient Electrodes for Supercapacitors. Journal of Physics. Conference Series, 1082, Article ID: 012081.

[18] Boechler, G.P., Whitney, J.M., Lent, C.S., Orlov, A.O. and Snider, G.L. (2010) Fundamental Limits of Energy Dissipation in Charge-Base Computing. Applied Physics Letters, 97, Article ID: 103502. https://doi.org/10.1063/1.3484959

[19] Pasha, M.S. and Prabhakar, G.C. (2015) Micro Controller Based Energy Efficient Four Steps Charging of a Capacitor Using a Buck Converter with Consecutive Changes of its Duty Ratio. International Journal of Engineering Trends and Technology, 27, 89-93. https://doi.org/10.14445/22315381/IJETT-V27P215

[20] Savitha, S. M., Rajani, H. P/ and Hunagund, S. M. (2018) Implementation of Low Power Adiabatic SRAM. International Journal of VLSI Design \& Communication Systems, 9, 1-18. https://doi.org/10.5121/vlsic.2018.9301

[21] Nakata, S. and Katagiri, Y. (2007) Electrostatic Energy, Potential Energy, and Energy Dissipation for a Width-Variable Capacitor System during Adiabatic Charging. Journal of Applied Physics, 101, Article ID: 034911. https://doi.org/10.1063/1.2436835 


\section{List of Symbols and Variables with Their Definitions}

$R$, Resistance;

$C$, Capacitance;

$N$, Number of steps;

$V_{0}$, Power supply voltage;

$q$, Charge on the capacitor;

$U$, Stored energy in the capacitor;

$Q$, Heat energy dissipated;

$E$, Consumed energy;

$\tau$, Time constant. 